\title{
Don't Forget to Take Your Vitamins! A Case of Gastric Bypass Gone Wrong
}

\author{
Ariel D. Sindel ${ }^{1, *}$, Gilad Shapira ${ }^{2}$, Chelsea Olson ${ }^{1}$, Derick Jenkins ${ }^{1}$ \\ ${ }^{1}$ Department of Internal Medicine, Virginia Commonwealth University Health Systems, Richmond, VA \\ ${ }^{2}$ Department of Internal Medicine, University of South Albama Medical Center, Mobile, AL \\ *Corresponding author: ariel.sindel@vcuhealth.org
}

\begin{abstract}
Gastric bypass, an increasingly common operation performed due to rises in obesity has greatly improved patient's quality of life along with health. This case report presents a 62 year old female three decades post Roux-En-Y gastric bypass who came in with shortness of breath. Knowing the malabsorptive nature of her weightloss surgery we suspected a nutritional deficiency and performed a full panel. Though we found multiple vitamin abnormalities, we were most concerned about her deficient thiamine levels. The complete workup led to a diagnosis of wet beriberi, a condition commonly seen in underdeveloped nations. With the rise in weight loss surgery, understanding and having a high clinical suspicion for such patients is necessary.
\end{abstract}

Keywords: Bariatrics, nutrition, beriberi, thiamine, vitamin, deficiency

Cite This Article: Ariel D. Sindel, Gilad Shapira, Chelsea Olson, and Derick Jenkins, "Don't Forget to Take Your Vitamins! A Case of Gastric Bypass Gone Wrong." American Journal of Medical Case Reports, vol. 5, no. 5 (2017): 119-121. doi: 10.12691/ajmcr-5-5-4.

\section{Introduction}

Recent data shows that more than one-third of adults in the United States are obese. With the prevalence of morbid obesity increasing by nearly $70 \%$ between 2000 and 2010. In 2015, there were roughly 196,000 bariatric surgeries performed. Though the gastric sleeve and lap-band are gaining popularity, the gold standard is still the Roux-En-Y gastric bypass [5]. Overall, weight loss is more quickly obtained in the RYGB population due to the malabsorptive nature of the surgery, with the trade-off being higher rates of nutritional deficiencies [3]. Though there is appeal to the rapid weight loss seen with RYGB in the first few months post-op in patient morbidities along with physical weight reduction, these added benefits come with some increased risk.

\section{Case}

A 62 year old female with newly diagnosed congestive heart failure, prior MI, hypertension and a Roux-en-Y gastric bypass 30 years prior who presented from for shortness of breath and further management of a new diagnosis of pulmonary hypertension and hypocalcemia. She reported complaints of muscle cramps of 1 week duration, diffuse weakness, poor appetite secondary to dysgeusia of one month duration, hair loss and thinning, scaly skin, easy bruising and visual disturbances. In addition since her bypass surgery, she had roughly $200 \mathrm{lb}$ weight loss since the surgery including $70 \mathrm{lbs}$ within the last month. Physical exam was notable for, glossitis, jugular venous distention to the angle of the jaw, a S4 gallop on cardiac multiple hyper and hyperpigmented macules with excoriations and scabbing on the bilateral arms (Figure 1), dry scaling skin on bilateral legs (Figure 2), dysmetria and mild dysdiadochokinesia. On labs, it was noted she had a hemoglobin of 11.0 with a MCV of 103.6, calcium of 6.0, Magnesium 1.2, intact PTH was 847, Vitamin D was 6.2. Her iron panel was normal, her B12 and RBC folate was also normal. However, given the concern for multiple micronutrient deficiencies, vitamin levels were obtained. Thiamine was 24 (normal 66), Vitamin $\mathrm{C}$ was 0.1, Zinc was 34, Selenium was 55, Copper 63, Vitamin A 7, Manganese was undetectable, and Vitamin E was 3.0. She received a TTE which showed a RV-RA gradient of 82 with an elevated RVSP of 87. Mild diastolic dysfunction with a MV E/A of 0.76 and an EF of 70-75\%. On CTA, she had a severely dilated pulmonary artery at $39 \mathrm{~mm}$ and cardiomegaly with four-chamber dilatation. The patient was aggressively repleted with high dose thiamine at $500 \mathrm{mg}$ every 8 hours for 4 days and transitioned to $100 \mathrm{mg}$ daily. She was also given aggressive repletions of the rest of her micronutrients. Prior to discharge the patient's dyspnea normalized along with her $\mathrm{O} 2$ saturation. Upon further investigation, it had appeared that she was compliant with her multivitamins up until 3 years prior to presentation. She was advised to begin intake higher levels of multivitamins but was unfortunately lost to follow up. 


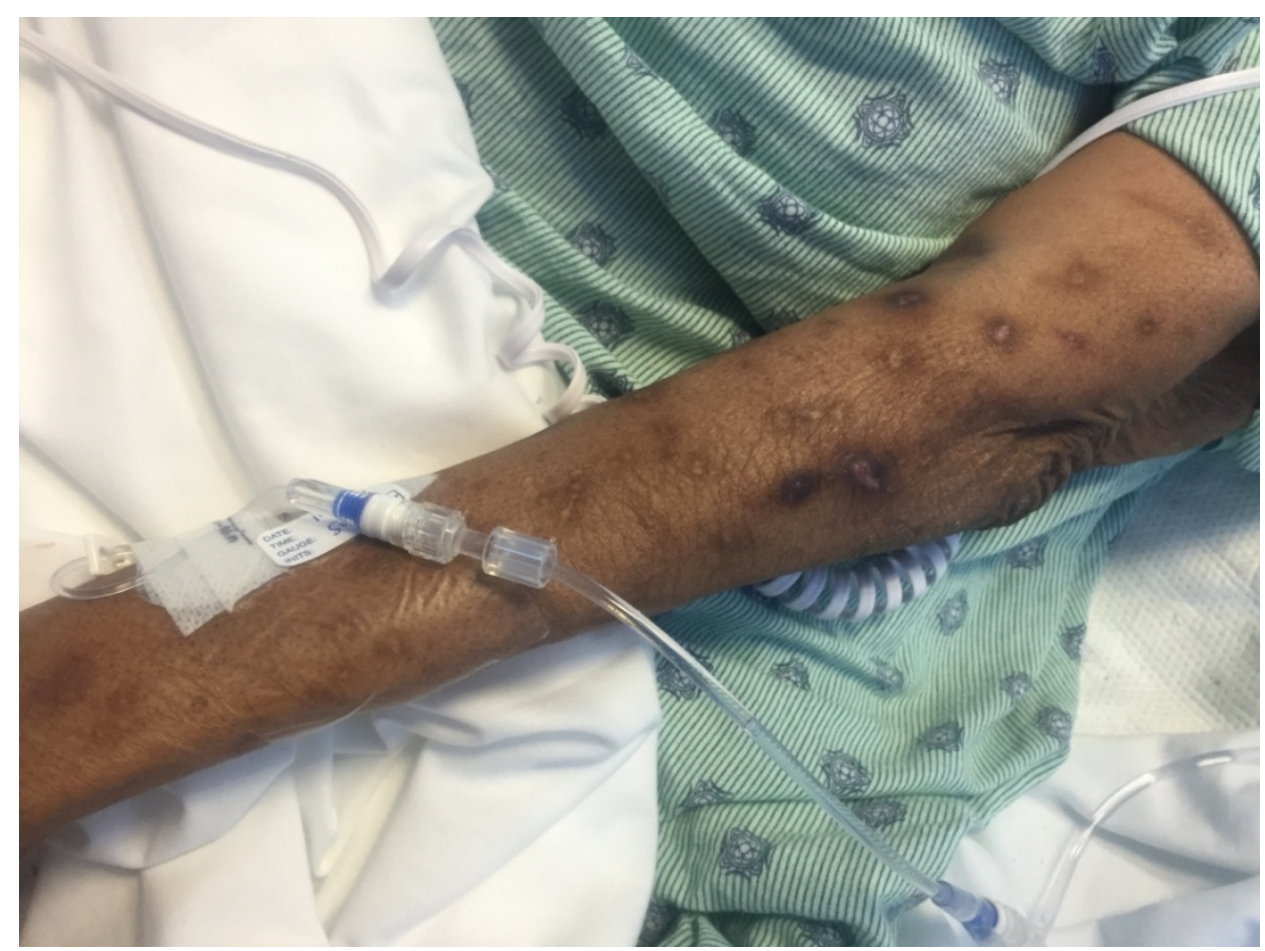

Figure 1. Hyperpigmented macules with excoriations along the upper extremity attributable to her multiple vitamin deficiencies

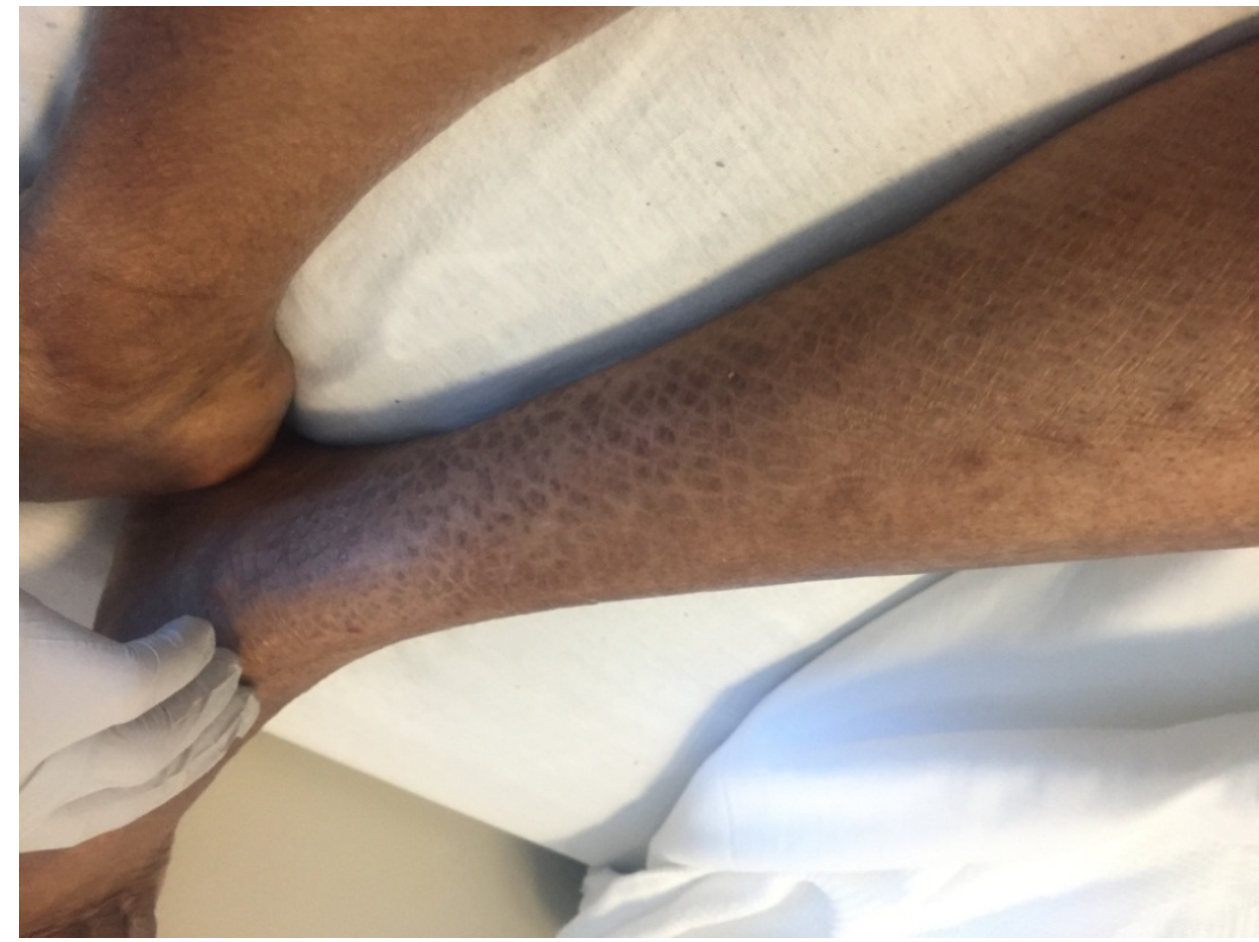

Figure 2. Icthyosis on bilateral lower extremities most likely representing Selenium Deficiency

\section{Discussion}

This patient presented with signs and symptoms concerning for multiple vitamin deficiencies which were then corroborated with laboratory evidence. The physical exam findings for vitamin deficiencies can often be subtle and overlooked, but it must always be high on the differential for someone who has done bariatric surgery. The most concerning was her thiamine deficiency. In the setting of edema, signs and symptoms of other deficiencies and an increased EF of $70-75 \%$ lead us towards the diagnosis of wet beriberi. Wet beriberi is not commonly seen in the USA, however, it is an important diagnosis to keep in mind, especially with high output heart failure having a high mortality rate. With the United States population becoming more obese, bariatric surgeries are on the rise, bringing along previously uncommon vitamin and nutrient deficiencies. Thiamine specifically has been previously been noted to be deficient in $15.5 \%$ of obese patients prior to surgery, with a greater predisposition in the female population [2]. Due to the possibly detrimental side effects of thiamine deficiency, multivitamin therapy with a thiamine component is recommended to be initiated 15 days prior to surgery [1]. 
Though many of the common nutrient deficiencies seen in bariatric surgery can pose long term danger to the patient, the troublesome reversibility of Wernicke's encephalopathy and Beriberi are an acutely imminent threat. With gastric banding gaining popularity as a weight loss option, less chronic nutrient deficiencies are expected due to the restrictive component of the technique in contrast to the malabsorptive RYGB. This stated, acute deficiencies can still be seen secondary to emesis most commonly due to the band tightening portion of the gastric binding procedure [3]. In all these post-surgical weight loss patient populations, clinicians must have a high clinical suspicion even for minor derangements in patient symptomology, in particular because RYGB is still the gold standard in surgical weight loss options. Labs such as Total Protein, Albumin, Pre-Albumin, Iron, Thiamine, B12, Folate, Calcium, Vitamin D, and Vitamin A should be monitored regularly. Though literature shows multiple patterns of monitoring, every three months for the first year, every six months for the second year, and every nine months in the third year appears to be a baseline [4]. In our case the patient began to neglect her multivitamin intake three decades after her RYGB and thus presented in such a manner.

\section{Conclusions}

Bariatric surgery is a growing field largely due to the obesity epidemic. Nutritional deficiencies are one of the most commonly seen undesired issues with these procedures requiring careful monitoring for the remainder of the patient's lifetime. Though the benefits of weight-loss surgery have proven to be immense, it is important for patients to understand risks and responsibilities prior to surgery. It is important for clinicians to explain to patients that such an operation requires life-long diligence in nutrition and added supplementation.

\section{Statement of Competing Interests}

There was no financial support or competing interests.

Consent was given by the patient for us of the case in a case report.

\section{References}

[1] Aron-Wisnewsky J, Verger EO, Bounaix C, Dao MC, Oppert J-M, Bouillot J-L, et al. Nutritional and Protein Deficiencies in the Short Term following Both Gastric Bypass and Gastric Banding. PLoS One. 2016; 11(2): e0149588.

[2] Carrodeguas L, Kaidar-Person O, Szomstein S, Antozzi P, Rosenthal R. Preoperative thiamine deficiency in obese population undergoing laparoscopic bariatric surgery. Surg Obes Relat Dis Off J Am Soc Bariatr Surg. 2005. December; 1(6): 517-22; discussion 522.

[3] Ledoux S, Msika S, Moussa F, Larger E. Comparison of nutritional consequences of conventional therapy of obesity, adjustable gastric banding, and gastric bypass. Obes Surg. 2006 Aug; 16(8):1041-9.

[4] Sawaya RA, Jaffe J, Friedenberg L, Friedenberg FK. Vitamin, mineral, and drug absorption following bariatric surgery. Curr Drug Metab. 2012; 13: 1345-55.

[5] Sturm R., Hattori A. Morbid obesity rates continue to rise rapidly in the United States. Int. J. Obes. 2013 Jun; 37(6): 889-891. 